Website: http://revistas.lamolina.edu.pe/index.php/acu/index

(C) Universidad Nacional Agraria La Molina, Lima - Perú

\title{
Crecimiento económico y desnutrición crónica infantil regional en el Perú: Una aplicación del modelo de probabilidad en el período 2000-2016
}

\author{
Economic growth and chronic child malnutrition in Peruvian regions: An application of the \\ probability model for 2000-2016
}

\author{
Carlos Alberto Minaya Gutierrez ${ }^{1 *}$ \& Alizi Rosario Sánchez Macedo² \\ *Autor de correspondencia
}

\begin{abstract}
Resumen
El objetivo del presente estudio es analizar la relación de causalidad entre el crecimiento económico regional y la reducción de la desnutrición crónica infantil (DCI) en Loreto y Moquegua. Para ello, en primer lugar, se empleó el Test de Granger para evaluar el sentido de causalidad entre ambas variables y se determinó que es el crecimiento económico que genera reducción de las tasas de DCI, en ambas regiones, y no al revés, por lo menos en el período de análisis. Finalmente, aplicando el modelo logit, se obtuvo el valor de los efectos marginales de las principales variables socioeconómicas que determinan la probabilidad de ocurrencia de que una región presente tasas de DCI en su población infantil menor de cinco años. De esta manera queda implícita que la variable dependiente en el modelo es la DCI. En ese sentido, son seis las variables que explican en buena medida el valor de esta probabilidad. Alguna de ellas son: PBI per cápita regional, nivel monetario de pobreza, acceso a agua potable y grado de educación de la madre. En este punto vale la pena resaltar que los resultados presentan diferencias significativas, en ambas regiones. Por ejemplo, cuando el crecimiento económico aumenta en $1 \%$, la probabilidad de que la tasa de DCI se reduzca en Loreto es de 2,68\%, mientras que en Moquegua este valor asciende a solo 0,14\%. Palabras clave: Desnutrición crónica infantil; crecimiento económico; causalidad entre variables temporales; modelo logit; probabilidad de ocurrencia.
\end{abstract}

\begin{abstract}
The objective of this study It was analyze the causality relation between regional economic growth and the reduction of chronic child malnutrition (DCI) in Loreto and Moquegua. For this, first, the Granger Test was used to evaluate the sense of causality between both variables and it determined that it is the economic growth that generates reduction of the rates of DCI, in both regions, and it's not the other way around, at least in the analysis period. Finally, applying the logit model, the value of the marginal effects of the main socioeconomic variables that determine the probability of occurrence that a region presenting DCI rates in its children population under five years was obtained. In this way it is implicit that the dependent variable in the model is the DCI. In that sense, there are six variables that explain to a large extent the value of this probability. Some of them are: GDP per capita regional, poverty level, access to drinking water and level of mother's education. At this point it is worth noting that the results show significant differences in both regions. For example, when the economic growth increases by $1 \%$, the probability of the DCI rate's decreasing in Loreto is $2,68 \%$, while in Moquegua this value rises only to $0,14 \%$. Keywords: chronic child undernutrition; economic growth; causality between temporary variables; logit model; probability of occurrence.
\end{abstract}

\section{Introducción}

Durante la presente década y la anterior, los principales indicadores macroeconómicos del país han mostrado una mejora significativa. En particular, el dinamismo de las cifras de crecimiento económico ha superado no solo al promedio de los países de la región, sino también a los registrados por el Perú en las últimas tres décadas. En el caso del crecimiento económico regional (PBI per cápita), la dinámica ha sido muy distinta: en el caso de regiones típicamente extractivas de recursos naturales, como Ancash y Pasco, por ejemplo, se puede observar que ambas decrecieron, en el período 2007-2014, a tasas del 0,2 y $1,7 \%$ respectivamente (Anexo 1 ).

La teoría señala que dicho crecimiento económico incrementa el volumen de recursos disponibles para los agentes económicos, los cuales, van a reflejarse en mayores ingresos para las familias y el gobierno. Estos mayores ingresos contribuirán con el desarrollo humano, en tanto se utilicen en rubros que contribuyan efectivamente con el incremento de las capacidades de la sociedad: salud y educación.

\footnotetext{
${ }^{1}$ Universidad Nacional Agraria La Molina, Lima, Perú. Email: cminaya@lamolina.edu.pe

${ }^{2}$ Escuela de Posgrado, Universidad Nacional Agraria La Molina, Lima, Perú. Email: alizisanchezm@gmail.com
} 
Por otro lado, respecto del impacto del capital humano en el crecimiento económico, siguiendo a Vásquez (2012), este se puede constatar a través de dos tipos de modelos; el primero que enfatiza el rol de la acumulación del capital humano - como factor de producción per se -, mientras que el segundo considera los impactos del nivel de capital humano en el crecimiento de la productividad a través de la capacidad de adaptación de tecnologías existentes e innovación. De esta manera, una mayor dotación de educación y salud de calidad redundará en un mayor crecimiento económico.

En base a lo anterior, la desnutrición crónica infantil es un problema de salud pública cuyas consecuencias se manifiestan a lo largo de todo el ciclo vital, y que afecta principalmente a los pobres y pobres extremos. Su principal efecto negativo inmediato (corto plazo) es la mayor probabilidad de ocurrencia de enfermedades o muerte prematura en niños menores de cinco años; mientras que en el largo plazo afecta el desempeño escolar, la capacidad de trabajo; y ocasiona costos y pérdidas económicas a las familias y el gobierno (Alcazar et al., 2013).

Siguiendo a Glewwe y King (2001) y Martinez et al. (2006), se conoce que los costos en productividad (Figura 1) asociados a la desnutrición crónica infantil provienen de $t$ res fuentes. La primera de ellas es la asociada a la deserción escolar provocada por el menor desarrollo cognitivo (repitencia, rezago, menor capacidad de atención). La segunda fuente es la asociada a la menor productividad, provocada por el menor desarrollo cognitivo que permanece como secuela de la desnutrición crónica infantil en la adultez (asociado a menores ingresos salariales por el bajo capital humano), mientras que la tercera es la asociada a las pérdidas de fuerza laboral
(PEA), como consecuencia de las muertes prematuras que se pueden asociar a la desnutrición crónica de los niños menores de cinco años.

Según la IDI (2016), aunque la situación actual de la desnutrición crónica infantil (DCI) en el país ha mejorado con los años, sigue siendo un gran problema que impacta no solo al sector salud, sino también al desarrollo de la economía peruana. En ese sentido, la misma fuente señala

\section{Materiales y métodos}

\section{Test de Granger}

Es importante señalar que la correlación entre variables no implica necesariamente una relación de causa en el sentido estricto de esta palabra. En este sentido, el planteamiento de Granger analiza la relación de causalidad de forma que se dice que la variable $\mathrm{Y}$ es causada por $\mathrm{X}$, si $\mathrm{X}$ contribuye a la estimación de $\mathrm{Y}$, o de forma equivalente si los coeficientes de la variable $\mathrm{X}$ (y su(s) rezago(s), si los hubiera) son significativos estadísticamente. No obstante, es importante resaltar que la afirmación $X$ causa a $Y$ no implica que $Y$ sea el efecto o el resultado de $\mathrm{X}$ solamente, pues intervienen, además, otros factores (variables) al margen de X (UAM, 2017). Así, a partir de dos variables temporales, $\mathrm{X}_{t}$ e $\mathrm{Y}_{\mathrm{t}}$ (Anexo 2); la prueba de causalidad supone que la información relevante para la predicción de dichas variables está contenida únicamente en su información pasada. La prueba implica la estimación de las siguientes regresiones (El principal supuesto es que ambas variables (X e Y) son estacionarias y que las perturbaciones y no están correlacionadas. Además de ello, es importante señalar que esta prueba es muy sensible a la longitud del rezago utilizado):

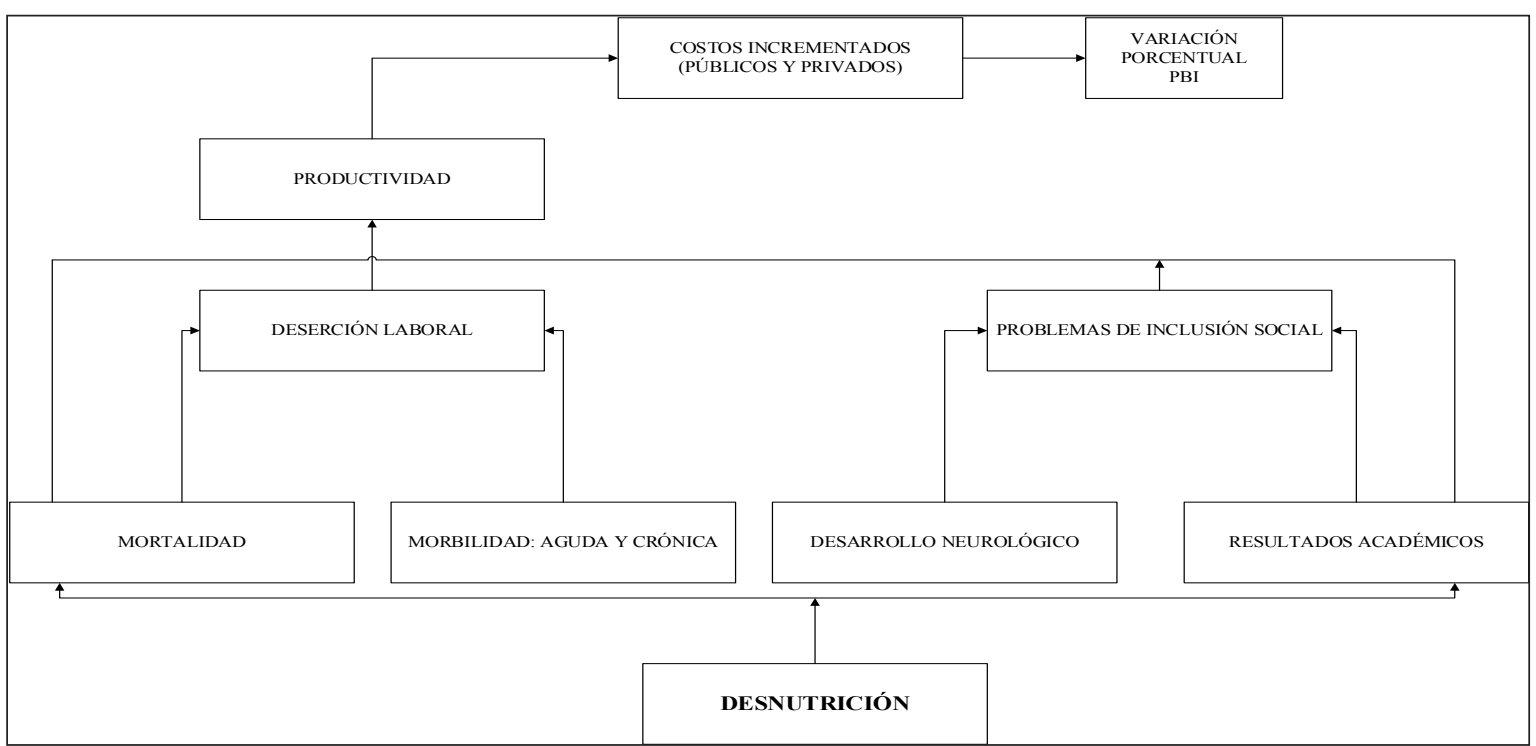

Figura 1. Consecuencias de la desnutrición crónica infantil

Fuente: Adaptado de CEPAL-UNICEF (2006). 


$$
\begin{aligned}
& \mathrm{Y}_{\mathrm{t}}=\sum_{\mathrm{i}=1}^{\mathrm{n}} \alpha_{\mathrm{i}} \mathrm{X}_{\mathrm{t}-\mathrm{i}}+\sum_{\mathrm{j}=1}^{\mathrm{n}} \beta_{\mathrm{j}} \mathrm{Y}_{\mathrm{t}-1}+\mathrm{u}_{1 \mathrm{t}} \cdot \ldots \text { (a) } \\
& \mathrm{X}_{\mathrm{t}}=\sum_{\mathrm{i}=1}^{\mathrm{n}} \mathrm{Y}_{\mathrm{t}} \mathrm{X}_{\mathrm{t}-\mathrm{i}}+\sum_{\mathrm{j}=1}^{\mathrm{n}} \delta_{\mathrm{j}} \mathrm{Y}_{\mathrm{t}-\mathrm{j}}+\mathrm{u}_{2 \mathrm{t} \cdots(\mathrm{b})}
\end{aligned}
$$

Apartir de las estimaciones anteriores, se pueden distinguir cuatro casos:

\begin{tabular}{|c|c|}
\hline $\begin{array}{l}\text { 1. La causalidad unidireccional } \\
\text { de X hacia Y es la indicada } \\
\text { si los coeficientes estimados } \\
\text { sobre la X rezagada en (a) son } \\
\text { estadísticamente diferentes de } \\
\text { cero considerados como grupo } \\
\text { y el conjunto de coeficientes } \\
\text { estimados sobre Y rezagado } \\
\text { en (b) no es estadísticamente } \\
\text { diferente de cero. }\end{array}$ & $\begin{array}{l}\text { 2. Al contrario, hay } \\
\text { causalidad unidireccional de } \\
\text { Y hacia X si el conjunto de } \\
\text { coeficientes de X rezagada } \\
\text { en (a) no es estadísticamente } \\
\text { diferente de cero y el } \\
\text { conjunto de coeficientes } \\
\text { de Y rezagado en (b) es } \\
\text { estadísticamente diferente } \\
\text { de cero. }\end{array}$ \\
\hline $\begin{array}{l}\text { 3. Se sugiere realimentación, } \\
\text { o causalidad bilateral, cuando } \\
\text { los conjuntos de coeficientes de } \\
\text { X y de Y son estadísticamente } \\
\text { significativos, diferentes de } \\
\text { cero. }\end{array}$ & $\begin{array}{l}\text { 4. Por último, se } \\
\text { sugiere independencia } \\
\text { cuando los conjuntos de } \\
\text { coeficientes de X y de Y } \\
\text { no son estadísticamente } \\
\text { significativos. }\end{array}$ \\
\hline
\end{tabular}

Tabla 1. Tipos de causalidad en el sentido de Granger

Fuente: Gujarati y Porter (2010).

Luego de establecer el tipo y dirección de causalidad, es importante señalar que dado que el futuro no puede predecir el pasado, si la variable X causa (a la manera de Granger) a la variable $\mathrm{Y}$, los cambios en $\mathrm{X}$ deben preceder a los cambios en Y. Por consiguiente, en una regresión de Y sobre otras variables, si incluimos valores rezagados de $\mathrm{X}$ y esto mejora significativamente la predicción de $\mathrm{Y}$, podemos decir que $\mathrm{X}$ causa a $\mathrm{Y}$ (a la manera de Granger) Los pasos comprendidos en la prueba de causalidad de Granger son los siguientes:

1. Realizar la regresión de $\mathrm{Y}$ actual $\left(\mathrm{Y}_{t}\right)$ sobre sus términos rezagados (definido previamente el tamaño del rezago) y otras variables, pero no incluir las variables $\mathrm{X}$ rezagadas en esta regresión, esta es la regresión restringida (Gujarati y Porter, 2010). A partir de esta regresión, obtener la suma de cuadrados residuales restringida, SCRR.

2. Efectuar la regresión con los términos rezagados de $\mathrm{X}$ (esta es la regresión no restringida) y obtener la suma de cuadrados residuales no restringida, SCRNR.

3. La hipótesis nula es $\mathrm{H}_{0}: \alpha_{\mathrm{i}}=0, \mathrm{i}=1,2, \ldots, \mathrm{n}$, es decir, los términos rezagados de $\mathrm{X}$ no pertenecen a la regresión.

4. Para probar esta hipótesis, se aplica la prueba F:

$$
\mathrm{F}=\frac{\left(\mathrm{SCR}_{\mathrm{R}}-\mathrm{SCR}\right.}{\mathrm{NR}) / \mathrm{m}}
$$

Que sigue la distribución $\mathrm{F}$ con $\mathrm{m} y(\mathrm{n}-\mathrm{k}) \mathrm{gl}$. En el presente caso, $\mathrm{m}$ es igual al número de términos rezagados de $\mathrm{X}, \mathrm{y} \mathrm{k}$ es el número de parámetros estimados en la regresión no restringida.

5. $\mathrm{Si}$ el valor $\mathrm{F}$ calculado excede al valor $\mathrm{F}$ crítico en el nivel seleccionado de significancia, se rechaza la hipótesis nula, en cuyo caso los términos rezagados de $\mathrm{X}$ pertenecen a la regresión. Esta es otra forma de decir que $\mathrm{X}$ causa a $\mathrm{Y}$.

6. Se repiten los pasos uno a cinco para probar el modelo (b), es decir, para definir si Y causa $\mathrm{X}$.

En la presente investigación primero se determinará el tipo y dirección de causalidad entre las variables: crecimiento económico (variación porcentual del PBI per cápita) y la tasa de DCI a nivel regional (también en su variación porcentual). Posteriormente, se utilizará la variable exógena determinada, conjuntamente con las demás variables socioeconómicas (nivel educativo de la madre, acceso a agua potable, ámbito de la vivienda, etc.) para explicar el comportamiento de la variable endógena (determinada) y de esta manera estimar también los efectos marginales de cada una de las variables independientes.

\section{Modelo de probabilidad: Logit}

A diferencia de los modelos de estimación econométrica donde la variable dependiente (Y) es cuantitativa y se tiene por objetivo estimar su valor esperado, dados los valores de las regresoras: $\mathrm{E}\left(\mathrm{Y}_{1 \mathrm{i}}\left|\mathrm{X}_{1 \mathrm{i}},\right| \mathrm{X}_{2 \mathrm{i}}, \ldots \mid \mathrm{X}_{\mathrm{Ki}}\right)$, donde las regresoras $(\mathrm{X})$ pueden ser cuantitativas como cualitativas. En los modelos donde $\mathrm{Y}$ es cualitativa (dicótoma $\mathrm{o}$ binaria; o policótoma en general), el objetivo es encontrar la probabilidad de que un acontecimiento suceda. Por otro lado, es importante mencionar que el modelo logit nace a partir del modelo lineal de probabilidad (MLP): $\mathrm{Y}_{i}=$ $\beta_{1}+\beta_{2}+\mathrm{u}_{\mathrm{i}}$, donde $\mathrm{Y}$ es la variable dependiente (binaria) y su esperanza condicional, $\mathrm{E}\left(\mathrm{Y}_{\mathrm{i}} \mid \mathrm{X}_{\mathrm{i}}\right)$, se interpreta como la probabilidad condicional de que el suceso tenga lugar dado $\mathrm{X}_{\mathrm{i}}$; es decir: $\operatorname{Pr}\left(\mathrm{Y}_{\mathrm{i}}=1 \mid \mathrm{X}_{\mathrm{i}}\right)$. Si se supone además que $E\left(e_{i}\right)=0$, esto es para obtener estimadores insesgados, se obtiene: $\mathrm{E}\left(\mathrm{Y}_{\mathrm{i}} \mid \mathrm{X}_{\mathrm{i}}\right)=\beta_{1}+\beta_{2} \mathrm{X}_{\mathrm{i}}$. Por último, si se denomina $\mathrm{p}_{\mathrm{i}}=$ probabilidad de que $\mathrm{Y}_{\mathrm{i}}=1$ (probabilidad de que ocurra el suceso), la variable $\mathrm{Y}_{\mathrm{i}}$ tiene la siguiente distribución de probabilidad:

\begin{tabular}{cc}
\hline $\mathrm{Y}_{\mathrm{i}}$ & Probabilidad \\
\hline 0 & $1-\mathrm{p}_{\mathrm{i}}$ \\
1 & $\mathrm{p}_{\mathrm{i}}$ \\
Total & 1 \\
\hline
\end{tabular}

Es decir, Yi sigue la distribución de probabilidad de Bernoulli. Entonces la esperanza matemática de $\mathrm{Y}_{\mathrm{i}}$ es: $\mathrm{E}$ $\left(Y_{i}\right)=0\left(1-p_{i}\right)+1\left(p_{i}\right)=p_{i}$. Por lo tanto al final se obtiene que la esperanza condicional de la ecuación original a estimar, en realidad se interpreta como la probabilidad condicional de $\mathrm{Y}_{\mathrm{i}}: \mathrm{E}\left(\mathrm{Y}_{\mathrm{i}} \mid \mathrm{X}_{\mathrm{i}}\right)=\beta_{1}+\beta_{2} \mathrm{X}_{\mathrm{i}}=\mathrm{p}_{\mathrm{i}}$.

$\mathrm{Si}$ bien es cierto que el MLP tiene la ventaja de ser de simple aplicabilidad, cuenta con algunos problemas de estimación, entre los cuales destacan a) la no normalidad del termino de error, b) heteroscedasticidad del término de error y c) la posibilidad de que la variable dependiente estimada Y, se encuentre fuera del rango 0-1. Sin embargo, Gujarati y Porter (2010) señalan que todos estos problemas 
son superables y que la principal razón por la cual el MLP no es un modelo muy atractivo empíricamente es que supone que $\mathrm{p}_{\mathrm{i}}=\mathrm{E}\left(\mathrm{Y}_{\mathrm{i}}=1 \mid \mathrm{X}_{\mathrm{i}}\right)$ aumenta linealmente con $\mathrm{X}$, es decir, el valor marginal o incremental de los coeficientes de $\mathrm{X}$ permanecen constantes para todo el dominio. En base a lo anterior, lo que se requiere es que el modelo probabilístico posea dos características: i) a medida que aumente $\mathrm{Xi}$, pi $=\mathrm{E}\left(\mathrm{Y}_{\mathrm{i}}=1 \mid \mathrm{X}_{\mathrm{i}}\right)$ también aumente, pero nunca se salga del intervalo 0-1 y ii) la relación entre $p_{i}$ y xi sea no lineal; lo que en palabras de Aldrich y Nelson (1984): se debe cumplir que "uno se acerca a cero con tasas cada vez más lentas a medida que se reduce $X_{i}$, y se acerca a uno con tasas cada vez más lentas a medida que $X_{i}$ se hace muy grande".

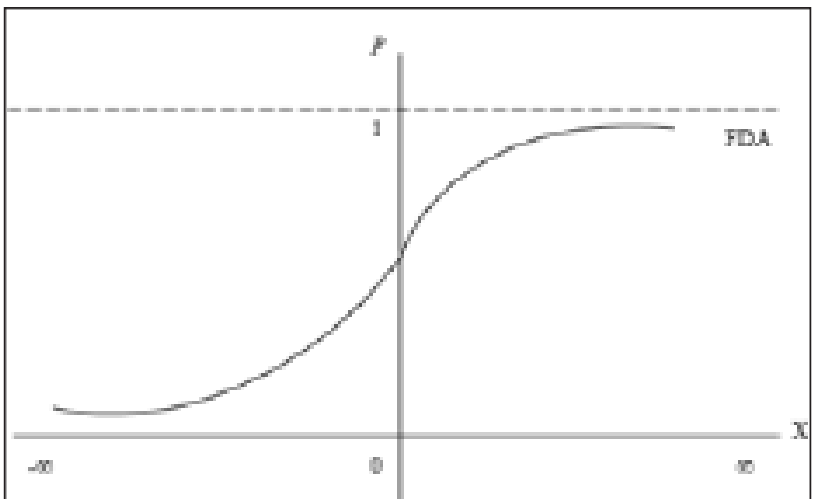

Figura 2. Función de distribución acumulativa (FDA) Fuente: Greene (1999).

La Figura 2 muestra la curva en forma de S o sigmoidea. Estorepresentalafuncióndedistribuciónacumulativa(FDA) de una variable aleatoria. Por consiguiente se puede utilizar fácilmente la FDA en regresiones de modelos en los cuales la variable de respuesta es dicótoma o binaria para adquirir valores de 0-1. Empíricamente Las FDA logística y normal son las que se seleccionan para representar los modelos logit y probit respectivamente (Cramer y Rider, 1991). Del modelo lineal de probabilidad se tiene que: $\mathrm{p}_{\mathrm{i}}=\beta_{1}+$ $\beta_{2} X_{i}$, donde $\mathrm{Xi}$ representa a las variables independientes y $\mathrm{p}_{\mathrm{i}}=\mathrm{E}\left(\mathrm{Y}_{\mathrm{i}} \mid \mathrm{X}_{\mathrm{i}}\right)$ representa la probabilidad de que suceda el evento. Considerando ahora la siguiente representación:

$$
P_{i}=\frac{1}{1+e^{-\left(\hat{\theta}_{1}+\beta_{2} x_{i}\right)}}
$$

Que reordenando los términos se obtiene:

$$
\mathrm{P}_{\mathrm{i}}=\frac{1}{1+e^{-z_{i}}}=\frac{e^{z_{i}}}{1+e^{z_{i}}}
$$

Donde: $Z_{i}=\beta_{1}+\beta_{2} X_{i}$. La ecuación anterior representa a la función de distribución logística acumulativa. De ahí se desprende fácilmente que si $Z_{i}$ se encuentra dentro de un rango de $-\infty \mathrm{a}+\infty, \mathrm{P}_{\mathrm{i}}$ se encuentra dentro del rango de 0 a 1 , y que $\mathrm{P}_{\mathrm{i}}$ no está linealmente relacionado con $\mathrm{Z}_{\mathrm{i}}$ (con) con lo cual hacen preferible este modelo respecto al MLP. Linealizando la ecuación anterior mediante el uso de la probabilidad de que no ocurra el evento: $1-\mathrm{P}_{\mathrm{i}}=\left(1 /\left(1+\mathrm{e}^{\mathrm{Zi}}\right)\right.$ se puede establecer la siguiente razón:

$$
\frac{B}{1-A^{2}}=\frac{1+e^{2}}{1+e^{-2}}=e^{5}
$$

Ahora $\mathrm{P}_{\mathrm{i}} /\left(1-\mathrm{P}_{\mathrm{i}}\right)$ es la razón de las probabilidades en favor de que ocurra un evento determinado. Finalmente, tomando el logaritmo natural de la ecuación anterior, se obtiene L (denominado logit), que no solo es lineal en $\mathrm{X}_{\mathrm{i}}$, sino también en los parámetros:

$$
L_{1}=\ln \left(\frac{R_{1}}{1-F_{1}}\right)-Z_{1}-\beta_{2}+\beta_{2} X_{1}
$$

\section{Logit con datos agrupados o duplicados: glogit}

A través del cálculo de $\hat{p}_{i}=n_{i} / N_{i}$ ), que representa la frecuencia relativa de casos de ocurrencia de un suceso (a lo largo del período: 2000-2016), por ejemplo: población regional menor de cinco años con desnutrición crónica, dado $\mathrm{X}_{\mathrm{i}}^{11}$ sobre la totalidad de observaciones; es decir, si con los datos disponibles se observa que para cada nivel de $X_{i}$, existe una determinada proporción que cumple la característica que se busca explicar (el suceso), entonces $\hat{p}_{i}$ se puede utilizar como una buena estimación de la verdadera Pi correspondiente a cada $\mathrm{X}_{\mathrm{i}}$.

En este caso, la variante del modelo logit permitiría trabajar con los datos disponibles, asumiendo, a priori ( $\sin$ corroborar aún con el Test de Granger), que la variable dependiente dicótoma (Y), que no es observada directamente en términos de 1 y 0 (total de la población de niños menores de cinco años con desnutrición crónica o no) sea la DCI.

Finalmente, lo que se calculará será el efecto marginal de cada una de las variables explicativas, agrupadas en $X_{i}$, en la ecuación (e), para obtener medidas cuantitativas del efecto marginal de cada una de las variables socioeconómicas en la probabilidad de que la región presente la condición de DCI en su población infantil menor de cinco años.

\section{Ámbito de la investigación}

Enestainvestigación se analizaron las regiones de Moquegua y Loreto. La primera, porque durante los últimos años cuenta con el PBI per cápita más elevado del Perú: pasó de S/. 45, 367 a S/. 47, 144 entre el 2007 y el 2014, además de ser la región que cuenta con un mayor porcentaje de cobertura de agua (SINAI, 2017) (componente de infraestructura asociada directamente con el nivel de bienestar social): $97,2 \%$ (al 2014) y la más baja proporción de su población menor de cinco años con condición de DCI. Por el contrario, Loreto es una de las regiones con menores ingresos en el Perú: pasó de S/. 7, 315 a S/. 8, 484, en el mismo período, y en el caso de acceso agua potable y de proporción de su población infantil con condición de DCI, en contraste de Moquegua, Loreto es la región con más bajo acceso a nivel nacional: 53,9\% para el caso de agua potable y $27,3 \%$ de población infantil con DCI, la más alta a nivel nacional. Respecto de su población, se puede comentar que si bien es cierto Loreto cuenta con ocho provincias; en los últimos 15 
años, el 74\% de su población regional se concentró en solo tres de ellas: Maynas (Iquitos), Alto Amazonas y Requena. Moquegua, por el contrario cuenta con solo tres regiones y el porcentaje de su población infantil (menores de cinco años) respecto de la población regional representó, en promedio del 2005 al 2015, el 8,1\%. En el caso de Loreto esta cifra es del $12,4 \%$.

\section{Resultados y discusión}

Si bien es cierto, existen antecedentes de investigación de CENAN - INS (2007); Velázquez (2009); Palomino (2010); Sifuentes (2012) y de Alcázar et al. (2013); en el caso peruano, que analizan la misma problemática y dejan evidencia que la desnutrición (crónica infantil, aguda y global) genera costos económicos asociados, no solo a la salud, sino a la educación y productividad; siendo a esta última a la que mayor efectos negativos conlleva (por lo que se discute aún en la literatura sobre el círculo vicioso: pobreza, desnutrición y baja productividad). Además de ello, en base a los antecedentes revisados, se ha podido identificar que las principales variables socioeconómicas que explican el grado de desnutrición son los ingresos, nivel de pobreza, PBI per cápita, grado de educación de la madre y acceso a agua y saneamiento principalmente. A nivel de antecedentes a investigación internacional, vale la pena resaltar el trabajo de The Lancet Global Health (2014) que analiza los patrones de crecimiento infantil en 36 países en desarrollo, en el período 1990 - 2011, y concluye que, contrariamente a la creencia generalizada, el crecimiento económico tiene poco o ningún efecto sobre el estado nutricional de los niños en los lugares más pobres del mundo. Los resultados muestran que un aumento de 5\% en el PIB per cápita se asoció con una pequeña reducción en las probabilidades de padecer retrasos del crecimiento $(0,4 \%)$, bajo peso $(1,1 \%)$ o estar desnutrido $(1,7 \%)$ (todas estas variables son signos de desnutrición infantil). En base a lo anterior, el aporte de la presente investigación, apunta a conocer con más precisión los efectos (marginales) de las principales variables socioeconómicas, mencionadas anteriormente, a nivel regional, y su impacto en la reducción de la DCI en su población infantil. Además, a partir de lo que la teoría económica señala, en el corto plazo, respecto del sentido unidireccional de la causalidad entre el crecimiento económico y la desnutrición, los resultados de este estudio brindarán elementos cuantitativos para que se puedan priorizar políticas públicas (económicas y sociales) de acuerdo a cada región. Es en ese sentido que esta investigación analiza dos regiones representativas del Perú.

\section{Loreto}

Analizando sus principales estadísticas, a nivel regional, así como los coeficientes de correlación y covarianzas (Anexo 3); en líneas generales, se puede comentar que el valor del PBI (en niveles) en esta región es uno de los más bajos a nivel nacional, creciendo en promedio (2000-2016) al 2,1\%, mientras que la DCI se redujo, en promedio, en el mismo período a 4,1\%. Respecto del grado de educación de la madre (Palomino, 2010), se puede comentar que esta región cuenta con un 10\% (promedio 2000-2016), una de las mayores tasas a nivel nacional.

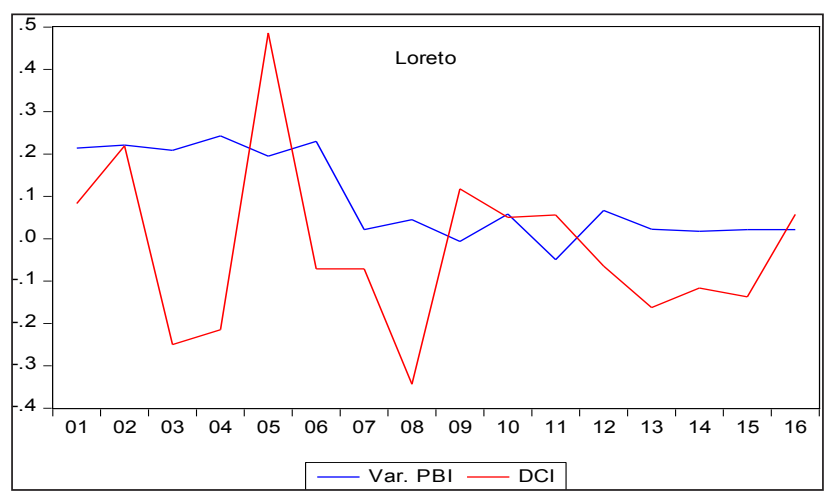

Figura 3. Variación del PBI y tasa de DCI en Loreto Elaboración propia en base al software E-views 8

\section{Moquegua}

Al igual que en el caso de Loreto, además del nivel medio del PBI, se analizaron sus principales estadísticas, a nivel regional, así como los coeficientes de correlación y covarianzas (Anexo 4). En líneas generales, se puede comentar que el valor de su PBI (el más alto a nivel nacional) creció, en promedio (2000-2016) al 0,6\%, mientras que la DCI se redujo, en promedio, en el mismo período a $7 \%$. La incidencia monetaria (pobreza total) promedio (2000-2016) es de $22,7 \%$, descendiendo a una tasa promedio anual de 5,36\%.

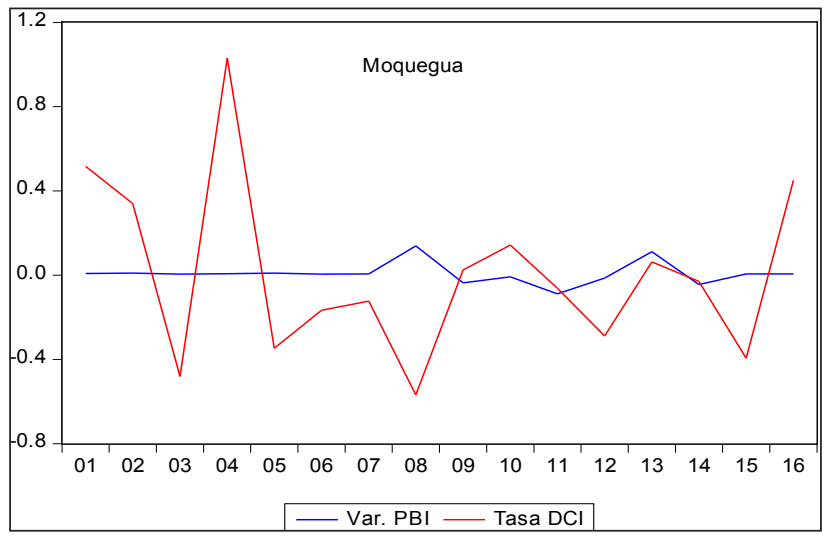

Figura 4. Variación del PBI y tasa de DCI en Moquegua Elaboración propia en base al software E-views 8

Por otro lado, se efectuó el Test de Granger, en ambas regiones, para determinar el tipo y dirección de causalidad entre las variables: Y: crecimiento económico (variación del PBI per cápita) y X: tasa de desnutrición crónica infantil (DCI). Se utilizaron las ecuaciones simultáneas (a) y (b), incluyéndose 1 rezago para la variable independiente y dos rezagos para la variable dependiente, pero considerando ambas variables de la siguiente manera $\mathrm{Z}_{\mathrm{t}}=\left(\Delta \mathrm{Y}_{\mathrm{t}}\right) / \mathrm{Y}_{\mathrm{t}} \mathrm{y} \mathrm{W}=$ $\left(\Delta X_{t}\right) / X_{t}$; y luego de determinada la dirección de causalidad 
se procederá a considerar las demás variables explicativas en el modelo: ecuaciones restringidas y no restringidas; incluyéndose dos rezagos de la variable dependiente (que se determinó previamente):

Primero: Sentido de causalidad (condición necesaria del Test de Granger).

$$
\begin{aligned}
& \mathrm{W}_{\mathrm{t}}=\gamma 1 \mathrm{Z}_{(\mathrm{t}-1)}+\gamma_{1} \mathrm{Z}_{(\mathrm{t}-1)}+\theta_{1} \mathrm{~W}_{(\mathrm{t}-1)} \\
& \mathrm{Z}_{\mathrm{t}}=\delta_{1} \mathrm{~W}_{(\mathrm{t}-1)}+\delta_{1} \mathrm{Z}_{(\mathrm{t}-2)}+\beta_{1} \mathrm{Z}_{(\mathrm{t}-1)}
\end{aligned}
$$

Segundo: Ecuaciones restringidas y no restringidas (condición suficiente del Test de Granger).

$$
\begin{aligned}
& \mathrm{W}_{\mathrm{t}}=\mu_{1} \mathrm{X}_{1}+\mu_{2} \mathrm{X}_{2}+\mu_{3} \mathrm{X}_{3}+\mu_{4} \mathrm{X}_{4}+\mu_{5} \mathrm{X}_{5} \ldots \text { Ec.restringida } \\
& \mathrm{W}_{\mathrm{t}}=\alpha_{4} \mathrm{Z}_{(\mathrm{t}-1)}+\mu_{1} \mathrm{X}_{1}+\mu_{2} \mathrm{X}_{2}+\mu_{3} \mathrm{X}_{3}+\mu_{4} \mathrm{X}_{4}+\mu_{5} \mathrm{X}_{5} \quad \text { Ec. no } \\
& \text { restringida }
\end{aligned}
$$

En ese sentido, la interpretación de los coeficientes estimados, en ambas regiones, es como sigue:

\section{Loreto:}

$\mathrm{W}_{\mathrm{t}}=-0,086 \mathrm{Z}_{(\mathrm{t}-1)}-0,076 \mathrm{Z}_{(\mathrm{t}-2)}+0,538 \mathrm{~W}_{(\mathrm{t}-1)}$

$(\mathrm{p}$-value $<5 \%)(\mathrm{p}$-value $<5 \%)(\mathrm{p}$-value $<5 \%)$

\section{Moquegua:}

$\mathrm{W}_{\mathrm{t}}=-0,094 \mathrm{Z}_{(\mathrm{t}-1)}-0,046 \mathrm{Z}_{(\mathrm{t}-2)}+0,318 \mathrm{~W}_{(\mathrm{t}-1)}$

$(\mathrm{p}$-value $<5 \%)(\mathrm{p}$-value $<5 \%)(\mathrm{p}$-value $<5 \%)$

- $\left(\partial \mathrm{W}_{\mathrm{t}}\right) /\left(\partial \mathrm{Z}_{(\mathrm{t}-1)}\right)=-0,086$; lo que en términos discretos es: $\left(\Delta \mathrm{W}_{\mathrm{t}}\right) /\left(\Delta \mathrm{Z}_{(\mathrm{t}-1)}\right)=-0,086$ : Cuando el PBI aumentó (un período atrás) en $1 \%(10 \%)$ anual $\left(\Delta \mathrm{Z}_{(\mathrm{t}-1)}\right)$, entonces la variación de la DCI $\left(\Delta \mathrm{W}_{\mathrm{t}}\right)$ en Loreto se redujo (después de 1 año) en $0,086 \%(0,86 \%)$.

- $\left(\partial \mathrm{W}_{\mathrm{t}}\right) /\left(\partial \mathrm{Z}_{(\mathrm{t}-2)}\right)=0,076 ;$ lo que en términos discretos es: $\left(\Delta \mathrm{W}_{\mathrm{t}}\right) /\left(\Delta \mathrm{Z}_{(\mathrm{t}-2)}\right)=0,076$ : Cuando el PBI aumentó (dos períodos atrás) en $1 \%(10 \%)$ anual $\left(\Delta \mathrm{Z}_{(\mathrm{t}-2)}\right)$, entonces la variación de la $\mathrm{DCI}\left(\Delta \mathrm{W}_{\mathrm{t}}\right)$ en Loreto se redujo (después de dos años) en $0,076 \%(0,76 \%)$.

- $\quad\left(\partial \mathrm{W}_{\mathrm{t}}\right) /\left(\partial \mathrm{W}_{(\mathrm{t}-1)}\right)=0,538$; lo que en términos discretos es: $\left(\Delta \mathrm{W}_{\mathrm{t}}\right) /\left(\Delta \mathrm{W}_{(\mathrm{t}-1)}\right)=0,538$ :

Cuando la DCI se redujo (un período atrás) en $1 \%$ $(10 \%)$ anual $\left(\Delta \mathrm{W}_{(\mathrm{t}-1)}\right)$, entonces la variación de la DCI $\left(\Delta \mathrm{W}_{\mathrm{t}}\right)$ en Loreto disminuyó (el período siguiente) en $0,538 \%(5,38 \%)$.

Luego de verificar el tipo y sentido de causalidad, se pasó a calcular, mediante el modelo logit, el valor de la probabilidad, a lo largo de la muestra, de que una región presente DCI: En líneas generales, se puede comentar existe un buen grado de ajuste (durante todo el período) de la probabilidad estimada respecto de la frecuencia relativa o proporción de la población infantil que presenta DCI respecto del total (p) en ambas regiones.

Tabla 2. Probabilidad estimada de que una región presente DCI en su población menor de cinco años

\begin{tabular}{ccccccc}
\hline \multirow{2}{*}{ Año } & \multicolumn{3}{c}{ Loreto } & \multicolumn{3}{c}{ Moquegua } \\
\cline { 2 - 7 } & $\mathrm{p}$ & $\mathrm{L}$ & $\begin{array}{l}\text { Prob. } \\
\text { estim }\end{array}$ & $\mathrm{p}$ & $\mathrm{L}$ & $\begin{array}{l}\text { Prob. } \\
\text { estim }\end{array}$ \\
\hline 2000 & 0,324 & $-0,73545$ & $34,9 \%$ & 0,093 & $-2,27754$ & $12,3 \%$ \\
2001 & 0,351 & $-0,61465$ & $34,5 \%$ & 0,141 & $-1,80701$ & $12,4 \%$ \\
2004 & 0,252 & $-1,08797$ & $33,5 \%$ & 0,199 & $-1,39256$ & $15,9 \%$ \\
2005 & 0,374 & $-0,51298$ & $35,6 \%$ & 0,130 & $-1,90096$ & $13,8 \%$ \\
2008 & 0,212 & $-1,31291$ & $25,4 \%$ & 0,041 & $-3,15232$ & $4,6 \%$ \\
2009 & 0,237 & $-1,1692$ & $25,2 \%$ & 0,042 & $-3,12718$ & $4,9 \%$ \\
2012 & 0,246 & $-1,12006$ & $22,4 \%$ & 0,032 & $-3,4095$ & $3,3 \%$ \\
2013 & 0,206 & $-1,34921$ & $20,6 \%$ & 0,034 & $-3,3468$ & $3,5 \%$ \\
2015 & 0,157 & $-1,68072$ & $16,9 \%$ & 0,020 & $-3,89182$ & $2,7 \%$ \\
2016 & 0,166 & $-1,61425$ & $15,2 \%$ & 0,029 & $-3,51103$ & $2,6 \%$ \\
\hline
\end{tabular}

Elaboración propia en base al software E-views 8

El valor de la probabilidad se estimó a partir de los valores corrientes de cada variable explicativa o independiente (ecuación g). En las siguientes ecuaciones, se muestra - para el año 2016 - dicho cálculo para Loreto y Moquegua respectivamente: $\mathrm{e}^{(3,9-0,0004(8851,1)+0,014(29,4)-0,022(10,6)-0,0076(64)-0,066(3}$
$2,1)-0,0058(28,9) / 1+\mathrm{e}^{(3,9-0,0004(8851,1)+0,014(29,4)-0,022(10,6)-0,0076(64)-0,066(32,1)-}$
$-0,0058(28,9))=15,2 \%$

$\mathrm{e}^{-22,59-8,84(47664,4)+0,059(7,1)-0,038(7,9)+0,134(98,4)+0,639(1}$ 9 , 7) - 0,105(31,2)/1+e $-22,59-8,84(47664,4)+0,059(7,1)-0,038(7,9)+0,133(98,4)+0,639(19,7)-$ $0,105(31,2))=2,55 \%$

Finalmente, a continuación se muestran los efectos marginales de las variables explicativas, sobre la probabilidad de que la región presente la condición de DCI en menores de cinco años.

Tabla 3. Efectos marginales (2016-2015) de las variables explicativas sobre la probabilidad de que una región varíe su condición de DCI en menores de cinco años

\begin{tabular}{cccccccc}
\hline \multicolumn{3}{c}{ Loreto } & \multicolumn{4}{c}{ Moquegua } \\
\hline V & Valor 1 & Valor 2 & D & V & Valor 1 Valor 2 & D \\
Y & 0,4249 & 0,3981 & $2,68 \%$ & Y & 0,0638 & 0,0624 & $0,15 \%$ \\
X1 & 0,1810 & 0,1692 & $1,17 \%$ & X1 & 0,0574 & 0,0551 & $0,24 \%$ \\
X2 & 0,1734 & 0,1745 & $-0,1 \%$ & X2 & 0,0611 & 0,0619 & $-0,008 \%$ \\
X3 & 0,4301 & 0,4164 & $-1,37 \%$ & X3 & 0,0642 & 0,0738 & $-0,96 \%$ \\
X4 & 0,4452 & 0,4611 & $-1,59 \%$ & X4 & 0,0550 & 0,0443 & $1,1 \%$ \\
X5 & 0,4314 & 0,6732 & $-24,1 \%$ & X5 & 0,0589 & 0,0645 & $-0,55 \%$ \\
\hline
\end{tabular}
Elaboración propia en base al software E-views 8

V: Variable

D:diferencial 
En el cuadro anterior se puede observar la variación (aumento o disminución, de acuerdo al diferencial) del valor de la probabilidad de que una región presente la condición de DCI en su población infantil, según la variable que se esté analizando: efectos marginales. Por ejemplo, en el caso de X1: porcentaje de la población considerada pobre (en términos monetarios), cuando se pasa de un nivel de 35\% a 29,4\% del año 2015 al 2016, en Loreto, se espera que los niños menores de 5 años puedan escapar de la condición de desnutridos crónicos en 1,17\%.

Del mismo modo, cuando X2: nivel de educación de la madre (medido como el porcentaje de mujeres mayores de 15 años que son analfabetas) desciende de 7,8 a 7,1\%, en Moquegua, se espera que los niños menores de 5 años sean más propensos a contraer la condición de desnutridos crónicos en $0,08 \%$, lo cual no corresponde con lo esperado, de acuerdo a la teoría económica. Lo anterior, debe ser tomado con cautela ya que se están analizando los coeficientes de manera parcial (ceteris paribus).

\section{Conclusiones}

$\mathrm{Si}$ bien es cierto, la literatura económica reconoce los efectos (impactos) entre el crecimiento económico y la desnutrición (crónica) a través de una doble causalidad; generalmente éstos se pueden diferenciar a corto y largo plazo: mayores tasas de crecimiento económico generan mejora en los diferentes indicadores sociales de la población, mientras que en el segundo caso, el problema de la desnutrición (crónica) ocasiona pérdidas en productividad (costos públicos y privados) y por ende reducen el crecimiento económico.

A partir de lo anterior, éste trabajo corroboró - debido a la muestra-que el crecimiento económico regional sí redujo las tasas de DCI (sentido de causalidad unidireccional). Esto a partir del cálculo del valor de la probabilidad de que una región peruana presente toda su población infantil con esta condición deficiente. Los resultados estimados -a partir del modelo logit -, para ambos casos, son significativos y con buena bondad de ajuste (predicción). Además se obtuvieron los efectos marginales de cada una de las seis variables explicativas donde resaltan los efectos marginales de la pobreza monetaria, además del crecimiento económico y el acceso a agua potable, en ambas regiones. En el caso de Loreto, vale destacar que la actividad extractiva de hidrocarburos tiene un efecto muy significativo: $24,1 \%$.

Finalmente, Ya que una de las principales variables que explican con mayor intensidad la reducción en la probabilidad de que una región presente la condición de DCI en su población menor de cinco años es el acceso a agua potable - asociado también al ámbito de la vivienda y el nivel de pobreza-, las políticas sociales deberían incidir en dotar de esta infraestructura social básica a nivel regional, provincial y distrital, ya que según los resultados de esta investigación, el impacto del aumento de cobertura de agua potable entre el año 2015-2016 (4,2\% y 1\% para el caso de Loreto y Moquegua respectivamente) redujo la probabilidad de que el total de población infantil presente la condición de DCI en 1,37\% y 0,96\% en ambas regiones respectivamente.

\section{Literatura citada}

Alcazar et al. 2013. Impacto económico de la desnutrición crónica, aguda y global en el Perú. Revista peruana de medicina experimental y salud pública 30 (4): 569-574.

Aldrich, J.; Nelson, F. 1984. Linear probability, logit, and probit models. Sage Publications 45.

CENAN [Centro Nacional de Alimentación y Nutrición]INSA [Instituto Nacional de Salud]. 2007. Efecto del nivel socioeconómico sobre algunos indicadores de salud y nutrición en la niñez.

CEPAL [Comisión Económica para América Latina y el Caribe] - UNICEF [United Nations International Children's Emergency Fund]. 2006. Desnutrición infantil en américa latina y el caribe. Desafíos: Boletín de la infancia y adolescencia sobre el avance de los objetivos de desarrollo del milenio (2): 1-12.

Cramer, J. y Rider, G. 1991. Pooling states in the multinomial logit model. Journal of econometrics, 47 (2), 267-272.

ENDES [Encuesta Demográfica y de Salud Familiar]. 2017. Disponible en: http://proyectos.inei.gob.pe/ endes/

Glewwe, O. \& King, E. 2001. The impact of early childhood nutritional status on cognitive development: Does the timing of malnutrition matter? The world bank economic review 15 (1): 81-113.

INEI [Instituto Nacional de Estadística e Informática]. 2015a. Indicadores regionales 2005 - 2016.

INEI. 2015b. Producto bruto interno por departamentos.

INEI. 2016a. estimaciones y proyecciones de población.

Gujarati, D. y Porter, D. 2010. Econometría. Quinta edición. Editorial Mcgraw-Hill, Ciudad de México, México. 248-254, 541-589, 652-669pp.

Greene, W. 1999. Análisis Econométrico. 3ra Edición. Editorial Prentice Hall, Caracas, Venezuela. 234$256 \mathrm{pp}$.

IDI [Iniciativa Contra la Desnutrición Infantil]. 2016. Desnutrición crónica y crecimiento económico. Disponibleen:http://www.iniciativacontradesnutricion. org.pe/idi-noticias-29-de-febrero-de-2016/

Kendall, M. \& Stuart, S. 1961. The advanced theory of statistics. Charles griffin publishers. New York, EEUU.

Martinez, R. et al. 2006. Modelo de análisis del impacto social y económico de la desnutrición infantil en américa latina. (CEPAL) s.l., s.e., 119.

Palomino, N. 2010. Análisis de equidad del nivel socioeconómico y la desnutrición crónica en niños menores de 5 años en el Perú. 2010: 85.

SINIA [Sistema Nacional de Información Ambiental]. 2017. Referencia consultada el 12/11/17: http://sinia. 
minam.gob.pe/indicador/1000

Sifuentes, E. 2012. Perú 1950-2010: Crecimiento económico y desnutrición crónica desde el desarrollo regional. Revista Investigaciones Sociales 16 (28): 5979.

The Lancet Global Health. 2014. Association between economic growth and early childhood nutrition Disponible en: https:/www.thelancet.com/journals/ langlo/a rticle/PIIS2214-109X(14)70268-2/fulltext

UAM [Universidad Autónoma de Madrid]. 2017. referencia consultada el 10/11/2017: https://www.uam. es/docencia/predysim/combinado6/6_3_we.htm

Vásquez, F. 2012. La relación entre crecimiento económico y desarrollo humano en el Perú. Revista moneda 151: 8-12.

Velásquez, A. 2009. Efectividad de programas de reducción de la pobreza en la nutrición infantil y los determinantes económicos de la desnutrición en países de escasos recursos. 26 (4): 15.

Anexo 1. Contribución al VAB extractivo nacional de las regiones del Perú y participación del PBI regional respecto al total nacional (precios constantes del 2007)

\begin{tabular}{|c|c|c|c|c|c|c|c|c|c|c|}
\hline Regiones & 2007 & 2008 & 2009 & 2010 & 2011 & 2012 & 2013 & 2014 & Media & $\% \mathrm{PBI} \mathrm{Nac}$, \\
\hline Ancash & 18,8 & 18,4 & 17,4 & 15,9 & 15 & 17,5 & 16,8 & 13,2 & 16,6 & 4,3 \\
\hline Cusco & 8,0 & 7,8 & 11 & 12,8 & 15,5 & 14,6 & 18,5 & 18,7 & 13,4 & 4 \\
\hline Arequipa & 9,9 & 11,3 & 10,9 & 11,4 & 11,4 & 10,8 & 10,1 & 8,8 & 10,6 & 5,2 \\
\hline Pasco & 9,1 & 8,1 & 7,2 & 6,3 & 6,0 & 6,0 & 5,5 & 6 & 6,8 & 1,3 \\
\hline Cajamarca & 5,4 & 6,5 & 7,6 & 6,7 & 6,6 & 6,9 & 5,9 & 5,3 & 6,4 & 2,6 \\
\hline Lima & 6,5 & 6,7 & 4,9 & 58 & 6,4 & 6,1 & 6,1 & 6,9 & 6,2 & 43,4 \\
\hline Moquegua & 6,5 & 6,4 & 6,5 & 6,1 & 5,0 & 5,2 & 49 & 5,1 & 5,7 & 2,1 \\
\hline Loreto & 5,3 & 5,0 & 4,9 & 5 & 3,8 & 3,9 & 4 & 3,8 & 4,4 & 2 \\
\hline Porcentaje agregado & 69,5 & 70,1 & 70,4 & 69,9 & 69,7 & 70,9 & 71,8 & 67,9 & 70 & 64,9 \\
\hline
\end{tabular}

Fuente: Elaboración propia en base a INEI (2015a)

Anexo 2. Variables que se emplearon en la investigación

\begin{tabular}{lccccccc}
\hline Año & $\mathrm{Y}$ & $\mathrm{X}$ & $\mathrm{X} 1$ & $\mathrm{X} 2$ & $\mathrm{X} 3$ & $\mathrm{X} 4$ & $\mathrm{X} 5$ \\
\hline 2000 & 6307,1 & 0,324 & 71,0 & 11,5 & 21,4 & 42,0 & 40,9 \\
2001 & 6442,1 & 0,351 & 70,0 & 11,1 & 23,0 & 41,4 & 40,0 \\
2002 & 6580,0 & 0,428 & 66,4 & 9,0 & 24,6 & 40,7 & 39,1 \\
2003 & 6720,8 & 0,321 & 68,4 & 10,2 & 26,3 & 40,1 & 38,3 \\
2004 & 6864,7 & 0,252 & 66,9 & 8,8 & 28,2 & 39,4 & 37,5 \\
2005 & 7011,6 & 0,374 & 75,0 & 9,2 & 30,2 & 38,8 & 36,7 \\
2006 & 7161,7 & 0,348 & 69,9 & 10,7 & 32,3 & 38,1 & 35,9 \\
2007 & 7315,0 & 0,323 & 57,5 & 12,2 & 34,6 & 37,5 & 35,1 \\
2008 & 7646,0 & 0,212 & 53,0 & 11,5 & 34,4 & 36,8 & 33,8 \\
2009 & 7596,0 & 0,237 & 54,7 & 11,6 & 46,3 & 36,2 & 33,2 \\
2010 & 8041,0 & 0,249 & 49,8 & 8,1 & 45,1 & 35,6 & 32,1 \\
2011 & 7644,0 & 0,263 & 48,1 & 10,1 & 40,7 & 35,0 & 25,8 \\
2012 & 8156,0 & 0,246 & 41,8 & 9,7 & 43,4 & 34,4 & 25,5 \\
2013 & 8338,0 & 0,206 & 37,4 & 7,7 & 51,0 & 33,8 & 26,0 \\
2014 & 8484,0 & 0,182 & 35,2 & 7,2 & 55,8 & 33,2 & 24,7 \\
2015 & 8665,6 & 0,157 & 35,0 & 11,0 & 59,8 & 32,7 & 29,5 \\
2016 & 8851,1 & 0,166 & 29,4 & 10,6 & 64,0 & 32,1 & 28,9 \\
\hline
\end{tabular}

Elaboración propia en base a INEI - ENDES (2017)

Nota: Y: PBI per cápita (S/. constantes 2007), X (tasa regional de DCI); X1: Pobreza total regional ; X2: Grado de educación de la madre; X3: Acceso fuentes de agua potable; X4: Ámbito regional de vivienda y X5: PBI per cápita extractivo. 
Anexo 3. Análisis de covarianzas y coeficientes de correlación en Loreto

\begin{tabular}{|c|c|c|c|c|c|c|c|}
\hline Correlation & $\mathrm{Y}$ & $X$ & $\mathrm{X} 1$ & $\mathrm{X} 2$ & $\mathrm{X} 3$ & $\mathrm{X} 4$ & $\mathrm{X} 5$ \\
\hline \multirow[t]{2}{*}{$\mathrm{Y}$} & 612312,8 & & & & & & \\
\hline & 1,000000 & & & & & & \\
\hline \multirow[t]{3}{*}{$\mathrm{X}$} & $-5082,784$ & 57,61612 & & & & & \\
\hline & $-0,855742$ & 1,000000 & & & & & \\
\hline & $-10793,41$ & 94,91143 & 209,8333 & & & & \\
\hline \multirow[t]{2}{*}{$\mathrm{X} 1$} & $-0,952215$ & 0,863196 & 1,000000 & & & & \\
\hline & $-302,6602$ & 1,471149 & 5,079099 & 2,052624 & & & \\
\hline \multirow[t]{2}{*}{$\mathrm{X} 2$} & $-0,269969$ & 0,135279 & 0,244734 & 1,000000 & & & \\
\hline & 9790,069 & $-82,73043$ & $-174,4730$ & $-4,228331$ & 165,3551 & & \\
\hline \multirow[t]{2}{*}{$\mathrm{X} 3$} & 0,972949 & $-0,847587$ & $-0,936660$ & $-0,229512$ & 1,000000 & & \\
\hline & $-2363,112$ & 19,42432 & 41,74055 & 1,107902 & $-37,91587$ & 9,327281 & \\
\hline \multirow[t]{2}{*}{$\mathrm{X} 4$} & $-0,988826$ & 0,837908 & 0,943503 & 0,253203 & $-0,965461$ & 1,000000 & \\
\hline & $-3679,735$ & 29,76459 & 68,30645 & 2,970671 & $-56,88604$ & 14,99363 & 28,16279 \\
\hline $\mathrm{X} 5$ & $-0,886119$ & 0,738908 & 0,888560 & 0,390717 & $-0,833603$ & 0,925106 & 1,000000 \\
\hline
\end{tabular}

Elaboración propia en base al software E-views 8

Anexo 4. Análisis de covarianzas y coeficientes de correlación en Moquegua

\begin{tabular}{|c|c|c|c|c|c|c|c|}
\hline Correlation & $\mathrm{Y}$ & $\mathrm{X}$ & $\mathrm{X} 1$ & $\mathrm{X} 2$ & $\mathrm{X} 3$ & $\mathrm{X} 4$ & $\mathrm{X} 5$ \\
\hline $\mathrm{Y}$ & $\begin{array}{l}5737047, \\
1,000000\end{array}$ & & & & & & \\
\hline X & $\begin{array}{l}-8168,142 \\
-0,617709\end{array}$ & $\begin{array}{l}30,47830 \\
1,000000\end{array}$ & & & & & \\
\hline $\mathrm{X} 1$ & $\begin{array}{l}-12175,05 \\
-0,441706\end{array}$ & $\begin{array}{l}54,45770 \\
0,857176\end{array}$ & $\begin{array}{l}132,4304 \\
1,000000\end{array}$ & & & & \\
\hline $\mathrm{X} 2$ & $\begin{array}{l}-2929,452 \\
-0,495927\end{array}$ & $\begin{array}{l}10,97210 \\
0,805879\end{array}$ & $\begin{array}{l}26,23749 \\
0,924493\end{array}$ & $\begin{array}{l}6,082043 \\
1,000000\end{array}$ & & & \\
\hline $\mathrm{X} 3$ & $\begin{array}{l}5928,678 \\
0,538011\end{array}$ & $\begin{array}{l}-19,59774 \\
-0,771593\end{array}$ & $\begin{array}{l}-46,72576 \\
-0,882552\end{array}$ & $\begin{array}{l}-9,014689 \\
-0,794518\end{array}$ & $\begin{array}{l}21,16630 \\
1,000000\end{array}$ & & \\
\hline $\mathrm{X} 4$ & $\begin{array}{l}-2603,616 \\
-0,572850\end{array}$ & $\begin{array}{l}8,479769 \\
0,809462\end{array}$ & $\begin{array}{l}19,18716 \\
0,878668\end{array}$ & $\begin{array}{l}3,693235 \\
0,789206\end{array}$ & $\begin{array}{l}-8,499723 \\
-0,973622\end{array}$ & $\begin{array}{l}3,600677 \\
1,000000\end{array}$ & \\
\hline X5 & $\begin{array}{l}-7848,535 \\
-0,592281 \\
\end{array}$ & $\begin{array}{l}24,65603 \\
0,807256 \\
\end{array}$ & $\begin{array}{l}55,24998 \\
0,867805 \\
\end{array}$ & $\begin{array}{l}10,57847 \\
0,775321 \\
\end{array}$ & $\begin{array}{l}-23,70370 \\
-0,931273 \\
\end{array}$ & $\begin{array}{l}10,30094 \\
0,981224 \\
\end{array}$ & $\begin{array}{l}30,60786 \\
1,000000\end{array}$ \\
\hline
\end{tabular}

Elaboración propia en base al software E-views 8 . 